\title{
Multiple programmed cell death pathways are involved in N-methyl-N-nitrosourea-induced photoreceptor degeneration
}

\author{
Miriam Reisenhofer • Jasmin Balmer • Rahel Zulliger • \\ Volker Enzmann
}

Received: 7 October 2014 / Revised: 5 December 2014 / Accepted: 16 December 2014 / Published online: 21 January 2015

(C) Springer-Verlag Berlin Heidelberg 2015

\begin{abstract}
Purpose To identify programmed cell death (PCD) pathways involved in N-methyl-N-nitrosourea (MNU)-induced photoreceptor (PR) degeneration.

Methods Adult C57BL/6 mice received a single MNU i.p. injection $(60 \mathrm{mg} / \mathrm{kg}$ bodyweight), and were observed over a period of 7 days. Degeneration was visualized by H\&E overview staining and electron microscopy. PR cell death was measured by quantifying TUNEL-positive cells in the outer nuclear layer (ONL). Activity measurements of key PCD enzymes (calpain, caspases) were used to identify the involved cell death pathways. Furthermore, the expression level of C/EBP homologous protein (CHOP) and glucose-regulated protein 78 (GRP78), key players in endoplasmic reticulum (ER) stress-induced apoptosis, was analyzed using quantitative real-time PCR.
\end{abstract}

Miriam Reisenhofer and Jasmin Balmer contributed equally to the project.

M. Reisenhofer $\cdot$ J. Balmer $\cdot$ V. Enzmann $(\triangle)$

Department of Ophthalmology, Inselspital, University of Bern,

Freiburgstrasse 14, 3010 Bern, Switzerland

e-mail: volker.enzmann@insel.ch

M. Reisenhofer

Paul Flechsig Institute for Brain Research, University of Leipzig,

Leipzig, Germany

M. Reisenhofer

Graduate School for Cellular and Biomedical Sciences, University of

Bern, Bern, Switzerland

\section{J. Balmer}

Nuffield Laboratory of Ophthalmology, Department of Clinical

Neurosciences, University of Oxford, Oxford, UK

R. Zulliger

Department of Cell Biology, University of Oklahoma Health

Sciences Center, Oklahoma City, OK, USA
Results A decrease in ONL thickness and the appearance of apoptotic PR nuclei could be detected beginning 3 days postinjection (PI). This was accompanied by an increase of TUNEL-positive cells. Significant upregulation of activated caspases $(3,9,12)$ was found at different time periods after MNU injection. Additionally, several other players of nonconventional PCD pathways were also upregulated. Consequently, calpain activity increased in the ONL, with a maximum on day 7 PI and an upregulation of CHOP and GRP78 expression beginning on day 1 PI was found.

Conclusions The data indicate that regular apoptosis is the major cause of MNU-induced PR cell death. However, alternative PCD pathways, including ER stress and calpain activation, are also involved. Knowledge about the mechanisms involved in this mouse model of PR degeneration could facilitate the design of putative combinatory therapeutic approaches.

Keywords Retinal degeneration · MNU · Photoreceptors · Mouse $\cdot$ Activity assays $\cdot$ PCD $\cdot$ Caspases $\cdot$ Calpain $\cdot$ ER stress

\section{Introduction}

The N-methyl-N-nitrosourea (MNU)-based animal model of retinal degeneration has been studied intensively [1]. The substance is an alkylating agent that causes guanine methylation and is known to induce different tumors $[2,3]$. It was also reported by Herrold that MNU causes the loss of photoreceptors (PR) [4]. Thereby, degeneration is mainly induced by apoptosis, as the inhibition of initiator and effector caspases protects the structures and functional properties of the PR [5, 6]. But caspase-independent apoptosis has also been shown in the MNU model [7]. Furthermore, macrophage infiltration corresponding to phagocytosis of degenerated PR and Müller cell 
proliferation to stabilize the damaged retina was found after MNU treatment [8]. Macrophage-dependent clearance is thereby fundamental to the maintenance of neural tissue in the process of apoptotic cell death [9]. However, only PRs undergo cell death without damaging further retinal tissue, including the retinal pigment epithelium (RPE) [10]. Different mechanisms have been described to induce PR apoptosis, including oxidative stress and the loss of retinal blood supply [11]. As retinitis pigmentosa (RP) is a human disease that is characterized by $P R$ loss leading to blindness, MNU-treated animals might serve as a model to investigate disease-associated features [12]. This could be especially true for RP forms that are caused by genesplicing mutations, which also share the characteristic of existing all over the body but affecting only the photoreceptors [13]. Another advantage, in addition to the specificity of PRs as target cells, is that MNU can be easily applied to all mouse strains without facing the necessity to intercross animals. Recent publications have provided more insight into the pathways involved in MNU-induced degeneration in the retina (see review [14]). Today, there is still a substantial amount of contradictory hypotheses concerning the trigger of MNUinduced cell death.

To investigate whether multiple cellular suicide mechanisms participate in the degeneration events in the MNU model, as described for other retinal degenerations [15], we have quantified the activity of key caspases of apoptotic pathways in retinal samples from MNU-treated mice and corresponding controls. In addition to the activation of caspase-3, as suggested by Yoshizawa et al. [5], we further investigated activation of caspases 9 and 12 . As the activation of calpains has been shown to trigger apoptosis in several animal models of retinal degeneration [16, 17], we have also assessed calpain-related cell death events after MNU treatment.

Endoplasmic reticulum (ER) stress has been implicated recently in a wide variety of human diseases, including PR degeneration [18]. Cellular stress conditions, such as perturbed calcium homeostasis and the accumulation of unfolded proteins, activate ER stress [19]. Several sensors of ER stress have been identified, including caspase-12. Because of the detected activation of an ER stress-specific caspase cascade, including caspases 3,9 , and 12 , we have additionally assessed the expression of glucose-regulated protein (GRP78) and C/EBP-homologous protein (CHOP), known as ER stress-related factors in photoreceptor degeneration [20].

The aim of this study was to gain more insight into the pathways of programmed cell death (PCD) involved in MNU-induced photoreceptor degeneration. Herein, we provide evidence that multiple PCD pathways are involved. Our results will facilitate further studies aiming to develop putative therapeutic approaches for retinal degenerative diseases, including combinatory treatment with multifaceted inhibitors.

\section{Methods}

MNU treatment $\mathrm{C} 57 \mathrm{BL} / 6$ mice (6-8 weeks old) received a single i.p. injection of sterile $1 \%$ MNU (Sigma-Aldrich, Buchs, Switzerland) in saline containing $0.05 \%$ acetic acid added immediately prior to injection. The final concentration of $60 \mathrm{mg} / \mathrm{kg}$ bodyweight and the investigated time points (d1, $\mathrm{d} 3$, and $\mathrm{d} 7$ ) post-injection (PI) were chosen in regard to previous trials that showed functional and histological changes at that dose and those time points [10]. Control animals received a similar volume of saline $(0.9 \% \mathrm{NaCl})$. No clinical signs of discomfort or weight loss were observed. The animals were treated according to the ARVO Statement for the Use of Animals in Ophthalmic and Vision Research, following approval by the commission for animal experimentation of the Canton of Bern, Switzerland.

Histology Eyes were fixed with $4 \%$ paraformaldehyde (PFA; MERCK, Darmstadt, Germany) in phosphate-buffered saline (PBS; Life Technologies, Zug, Switzerland) overnight, embedded in paraffin (Fisher Scientific, Rheinach, Switzerland), and $5-\mu \mathrm{m}$ sections were cut with a microtome (Leica RM2245; Biosystems, Muttenz, Switzerland). Sagittally oriented central sections at the level of the optic nerve head $(\mathrm{ONH})$ were stained with Mayer's hemalum and eosin (H\&E; Roth, Karlsruhe, Germany) and evaluated with NISElements (Nikon, Egg, Switzerland).

Electron microscopy Selected eyes (control, MNU) were fixed in Karnovksy solution (1 \% PFA, $3 \%$ glutaraldehyde, $3 \%$ sodium cacodylate- $\mathrm{HCl}$; Science Services, Munich, Germany) for at least $24 \mathrm{~h}$ before the lens was removed, after which the eyes were fixed for an additional $24 \mathrm{~h}$. The eyecups were washed with EM buffer ( $2.5 \%$ glutaraldehyde and $0.1 \mathrm{M}$ sodium cacodylate- $\mathrm{HCl}$ ) and then postfixed in $4 \%$ osmium tetroxide (Science Services). The tissue was dehydrated, washed with a resin/1,2-propylene oxide mixture (MERCK) and mounted in resin (ERL 4221, DER 736, NSA, and DMAE), mixed according to the manufacturer's instructions (Science Services). The resin blocks were trimmed and semithin sections $(1 \mu \mathrm{m})$ were cut. Overview staining with $0.5 \%$ toluidine blue (Sigma) was performed to assure sampling on the level of the ONH. Ultrathin sections $(80 \mathrm{~nm})$ were then cut, contrasted with $0.1 \%$ lead citrate (Sigma-Aldrich) and then visualized on a CM 12 electron microscope (EM; Philips Applied Technologies, Eindhoven, The Netherlands).

TUNEL staining Eyes were fixed with $4 \%$ PFA at $4{ }^{\circ} \mathrm{C}$ overnight, embedded in paraffin, and $5-\mu \mathrm{m}$ sections were cut. To assess cell death, terminal deoxynucleotidyl transferase deoxyuridine triphosphate nick-end labeling (TUNEL) was performed using the In Situ Cell Death Detection Kit, 
TMR red (Roche Diagnostics, Rotkreuz, Switzerland) according to the manufacturer's instructions. Nuclei were counterstained with 4',6-diamidino-2-phenylindole (DAPI, NucBlue ${ }^{\circledR}$ Fixed Cell ReadyProbes ${ }^{\circledR}$ Reagent, Life Technologies) and slides were mounted with ProLong ${ }^{\circledR}$ Gold Antifade Mountant (Life Technologies). Staining was visualized with a scanning laser microscope (Zeiss LSM710; Carl Zeiss Microscopy, Jena, Germany). TUNEL positive cells were counted in visual fields $(n=9$ per animal) of $61.14 \mathrm{~mm}^{2}$ in the area of the posterior pole close to the optic nerve head.

Activity assays Calpain as well as caspase-3, -9 and -12 activity assays (BioVision, Milpitas, CA, USA) were performed on retinal lysates according to the manufacturer's instructions. Briefly, retinas $(n \geq 4)$ were dissected, pooled, and lysed in RIPA buffer $\left(150 \mathrm{mM} \mathrm{NaCl}, 1.0 \%\right.$ IGEPAL $^{\circledR}, 0.5 \%$ sodium deoxycholate, $0.1 \%$ SDS, $50 \mathrm{mM}$ Tris, pH 8.0; SigmaAldrich) supplemented with a cocktail of inhibitors to prevent protein degradation (Complete Mini; Roche). Cell lysates were analyzed using the DC Protein Assay (Bio-Rad, Cressier, Switzerland), and aliquots of $50 \mu \mathrm{g}$ were introduced per assay. The samples were incubated for $1 \mathrm{~h}$ with the specific substrate, which emits a yellow-green fluorescence upon cleavage in the presence of the corresponding caspase or calpain protease. The cleaved substrate was then fluorometrically measured at $505 \mathrm{~nm}$ (Infinite 200PRO; Tecan, Männedorf, Switzerland). Activity data from MNUtreated animals were plotted against control values obtained from $\mathrm{NaCl}$-injected mice for each specific time point PI. Furthermore, three independent retinal lysate samples of MNU-treated mice were supplemented with $100 \mu \mathrm{M}$ of the calpain inhibitor Z-LLY-FMK (BioVision) before incubation in the substrate for $1 \mathrm{~h}$, in order to confirm a specific activity after MNU treatment.

In situ activity assay Calpain activity was measured using the cell-permeable fluorescent substrate tert-butoxycarbonyl-Lmethionineamide-7-amino-4-chloromethylcouarim (t-Boc-LeuMet, CMAC, Molecular Probes, Inc., Eugene, OR, USA) [16]. Briefly, unfixed retinal cryosections obtained from MNU-treated and control mice were incubated in calpain reaction buffer (CRB: $25 \mathrm{mM}$ HEPES, $65 \mathrm{mM} \mathrm{KCl}, 2 \mathrm{mM} \mathrm{MgCl} 2,2 \mathrm{mM}$ DTT, $1.5 \mathrm{mM} \mathrm{CaCl}_{2}$; Sigma-Aldrich) at room temperature for $15 \mathrm{~min}$. This was followed by incubation with $2 \mu \mathrm{M}$ t-Boc-LeuMet dissolved in $\mathrm{CRB}$ at $37^{\circ} \mathrm{C}$ in the dark for $1 \mathrm{~h}$. After washing in TBS, the slides were fixed in $4 \%$ PFA and assessed for apoptotic cell death using the In Situ Cell Death Detection Kit, Fluorescein (Roche) as described above. After washing with Tris-buffered saline (TBS, Sigma-Aldrich), slides were mounted with VECTASHIELD Mounting Medium with DAPI (Vector Laboratories, Burlingame, CA, USA). Images were analyzed using a fluorescence microscope (Eclipse 80i, Nikon)
Quantitative RT-PCR Total RNA was isolated using the RNeasy Mini Kit (Qiagen, Hombrechtikon, Switzerland) according to the manufacturer's instructions. Three independent samples obtained from four pooled retinas were used for each condition. RNA quantity and quality were assessed with the Experion Automated Electrophoresis system (Bio-Rad). For cDNA synthesis and subsequent qRT-PCR, only RNA samples with an RNA quality indicator (RQI) $>7.0$ were used. cDNA was synthesized from $1 \mu \mathrm{g}$ of total RNA, using the iScript cDNA Synthesis Kit (Bio-Rad) according to the manufacturer's instructions. cDNA was purified with the PCR Purification Kit (Qiagen). qRT-PCR was performed using an iQ5 real-time PCR Detection System (Bio-Rad). The 25- $\mu$ l PCR reaction mix included $12.5 \mu \mathrm{l}$ of $1 \times$ iQ SYBR Green Supermix (Bio-Rad), $1 \mu \mathrm{l}$ of cDNA (125 ng), $1 \mu \mathrm{l}$ of each forward and reverse primer $(400 \mathrm{nM})$, and $9.5 \mu \mathrm{l}$ of $\mathrm{dH}_{2} \mathrm{O}$. The following primer pairs were used: Gapdh (NM_008084) forward 5'-AACTTTGGCATTGTGGAAGG-3' \& reverse 5'ACACATTGGGGGTAGGAACA-3'; Chop (NM_007837) forward 5'-CTGCCTTTCACCTTGGAGAC-3' \& reverse 5'CGTTTCCTGGGGATGAGATA-3' [21]; Grp 78 (NM_001163434) forward 5'-CCTGCGTCGGTGTGTTCA AG-3' \& reverse 5'-AAGGGTCATTCCAAGTGCG-3' [20]. The reactions were incubated in a 96-well optical plate for $3 \mathrm{~min}$ at $95^{\circ} \mathrm{C}$, followed by 50 cycles of $20 \mathrm{~s}$ at $95^{\circ} \mathrm{C}, 20 \mathrm{~s}$ at $58{ }^{\circ} \mathrm{C}, 30 \mathrm{~s}$ at $72{ }^{\circ} \mathrm{C}, 1 \mathrm{~min}$ incubation at $95^{\circ} \mathrm{C}$, and a final incubation of $1 \mathrm{~min}$ at $75^{\circ} \mathrm{C}$. Relative mRNA expression was calculated using GenEx software (MultiD Analyses, Gothenburg, Sweden). Cycle thresholds were normalized against the reference gene Gapdh. Expression data are presented as means $\pm \mathrm{SD}$ calculated against the control samples. Expression in control samples was set to ' 1 '.

Statistics For each time point and dosage, the mean $\pm \mathrm{SD}$ of at least three independent experimental measures were calculated. SigmaPlot statistical software (Version 12; Systat Software, Erkrath, Germany) or SPSS (IBM, Hampshire, UK) was used to assess the data. The Student's $t$-test or oneway ANOVA test with post-hoc all pairwise multiple comparison procedures (Tukey's or Holm-Sidak test) was applied and differences were considered statistically significant at $P \leq$ 0.05 .

\section{Results}

Photoreceptor cell death after MNU treatment

MNU treatment caused a dramatic decrease of ONL thickness over time, whereas the RPE appeared undisturbed. The panel shows representative images of the different time points investigated (Fig. 1). These specific effects could also be 

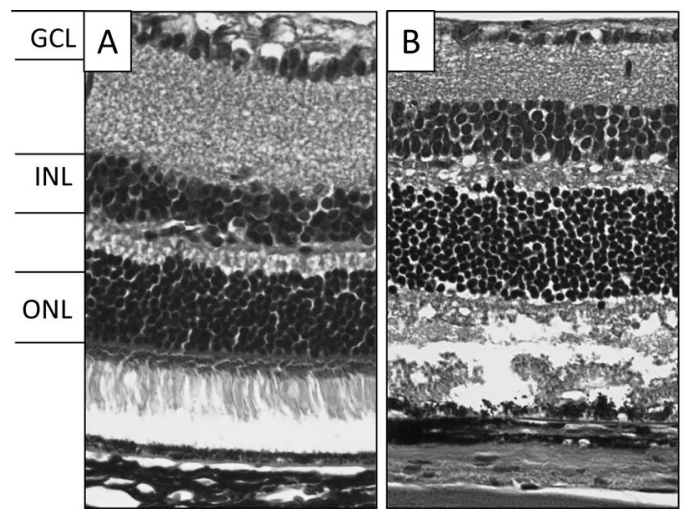

Fig. 1 Morphological changes in the retina after treatment with MNU $(60 \mathrm{mg} / \mathrm{kg})$ over time. Representative images of H\&E-stained retina sections showed significant changes in the sensory retina after the treatment (b-d) compared to the control (a). The panels show a decrease in the

visualized on the ultrastructural level, and were evident starting from day 3 PI (Fig. 2). The nuclei in the ONL displayed the hyperchromatic characteristics of apoptotic cell death (Fig. 2a). However, some nuclei had also changed shape, and displayed residual bodies and an altered chromatin structure (Fig. 2b), a characteristic of late apoptotic cells. At the same time point, the interface between photoreceptor cells and RPE appeared degenerated. The photoreceptor nuclei intermingled with the disturbed outer segments, and could be visualized adjacent to the RPE monolayer (Fig. 2c). The complete disappearance of the photoreceptor outer segments (Fig. 2d) was observed at the latest time point after treatment. At the same time point, the RPE layer still appeared healthy and displayed structures comparable to the control sample (Fig. 2e), indicating the direct and photoreceptor-specific effect of MNU. The treatment triggered PR cell death, as TUNEL positivity was only detectable within the ONL (Fig. 3b-d). The number of apoptotic cells was $4.0 \pm$ $1.8 \%$ on day $1 \mathrm{PI}$, reached the maximum with $60.4 \pm 14.6 \%$ on day 3 PI, and was still $49.5 \pm 14.5 \%$ on day 7 PI (Fig. 3e). No TUNEL-positive cells were found in the control samples (Fig. 3a).

PCD pathways in photoreceptor degeneration

To identify which cell death mechanisms were triggered by MNU, we investigated key molecules of caspase-dependent as well as caspase-independent pathways. MNU treatment led to an upregulation of the activity of several apoptotic caspases (Fig. 4). Whereas no increase in caspase-3 expression (17.8 \pm 8.1 RFU) was detectable at day 1 PI compared to the levels in control animals (15.8 $\pm 11.9 \mathrm{RFU})$, a significant increase was however detectable 3 days PI ( $72.6 \pm 32.0 \mathrm{RFU} ; P<0.001)$, as well as at day $7(51.1 \pm 11.4 ; P=0.02)$. The caspase activity was increased 5.8-fold and 3.7-fold respectively over the control values $(12.6 \pm 4.3,14.0 \pm 9.0)$. Besides the executer caspase-3, additional caspases were simultaneously upregulated after administration of MNU. Caspase-9 was
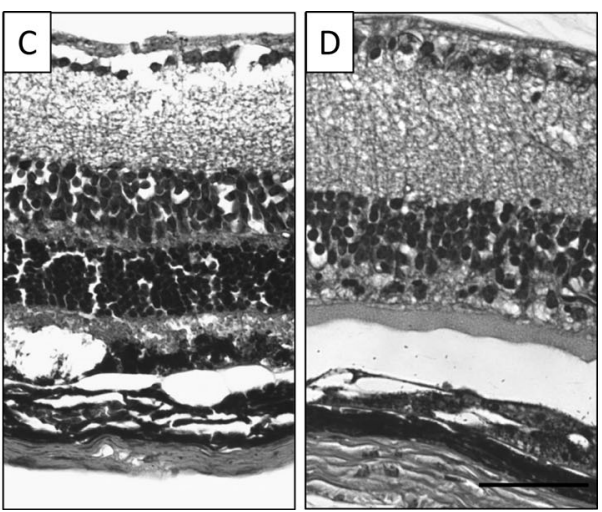

thickness of the ONL after treatment over time (control: a, day 1: b, day 3: c, day 7: d), whereas the RPE monolayer appears still intact, even at the latest time point investigated. The scale bar represents $100 \mu \mathrm{m}$

upregulated up to 4.6-fold at day $1 \mathrm{PI}$ in comparison to the value in control treated retinas $(115.2 \pm 44.7$ vs $26.3 \pm 17.1$ RFU; $P<0.01)$, whereas caspase-12 displayed a moderate but significant $(P=0.02)$ two-fold increase in expression at day 1 PI compared to the control values $(6737.4 \pm 1608.2$ vs $3285.8 \pm 1308.5 \mathrm{RFU}$ ). The activation of caspase- 3 confirmed the involvement of conventional apoptosis. However, other cell death mechanisms have to be addressed as caspaseindependent pathways might be involved as well.

Since in PR degeneration conventional cell death can be accompanied by the presence of activated calpains [22], we further assessed the activity of these calcium-dependent proteases directly in an in-situ activity assay. Retinas from MNUinjected mice displayed calpain-positive cells in the ONL on day 3 PI (Fig. 5a). Furthermore, a few TUNEL-positive cells in the ONL showed calpain activity (Fig. 5b), indicating that the MNU-induced PR cell death is at least partially caspaseindependent. No such cells were found in the same layer of the control samples (Fig. 5c). The obtained data were confirmed by a significant upregulation of activated calpain in protein lysates of retinas from MNU-treated animals compared to the controls at day 1 PI $(16,546 \pm 560$ RFU vs $11,804 \pm 1,669$ RFU; $P=0.03)$, at day 3 PI $(19,245 \pm 836$ RFU vs $14,042 \pm 1$, 664 RFU; $P=0.014)$ and at day 7 PI $(19,771 \pm 2,383 \mathrm{RFU}$ vs $13,379 \pm 2,099$ RFU; $P=0.002$; Fig. $5 \mathrm{~d})$. However, the upregulation was only moderate with a maximum increase of $47 \%$ at day 7 PI, and therewith smaller than the caspase-3 activation (see above). Incubation of the lysates with the calpain inhibitor Z-LLY-FMK prior to adding the calpain substrate led to a significant downregulation of calpain activity, by $54 \%$ (d3: $18,579 \pm 1,833 \mathrm{RFU}$ in MNU-treated samples vs $8,574 \pm 667$ RFU in the samples with the inhibitor).

Involvement of ER stress

MNU might also trigger activation of the ER stress pathway, as previously proposed [10]. The increased caspase- 9 and 

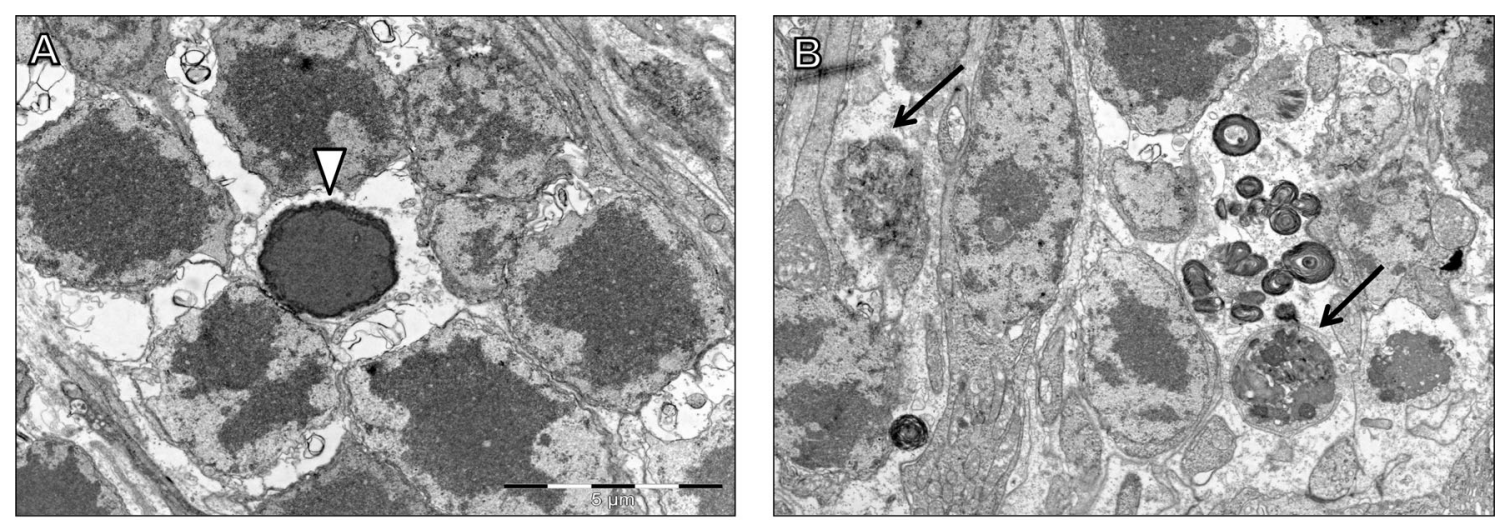
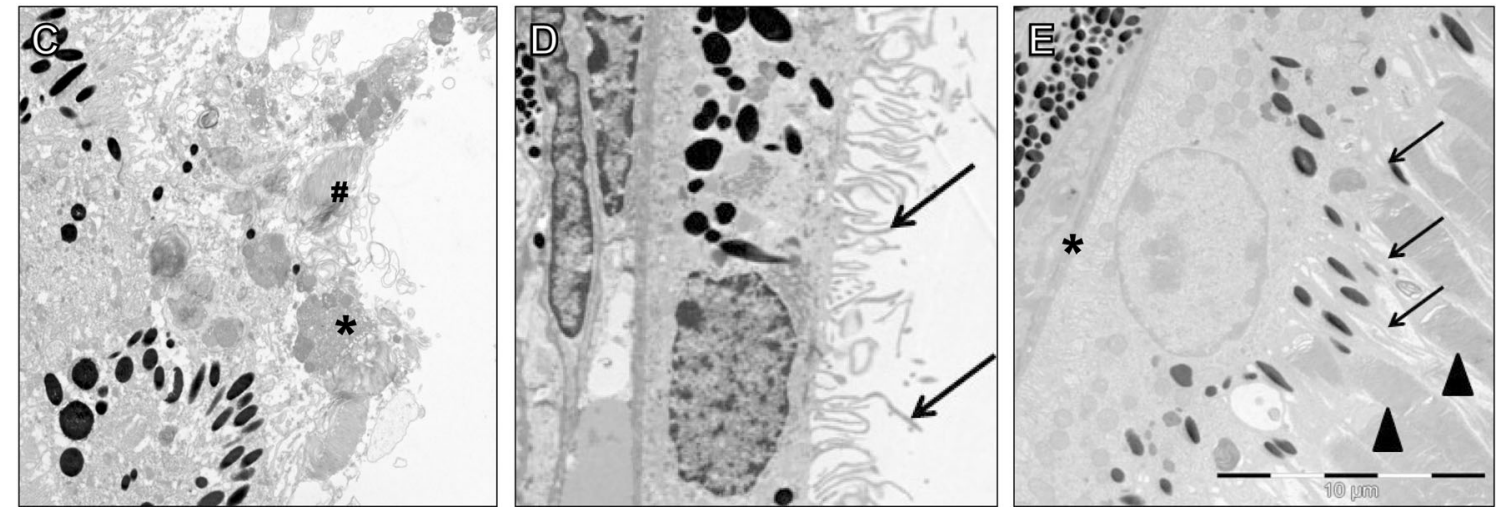

Fig. 2 Apoptotic nuclei (a; white arrowhead) appeared in the outer nuclear layer next to non-apoptotic nuclei 3 days after treatment with $60 \mathrm{mg} / \mathrm{kg}$ MNU. Nuclei with disordered but not fully condensed chromatin and apoptotic bodies (b; arrows) were found under the same conditions. At the same time point, the interface between photoreceptor cells and RPE was disturbed. Thereby, photoreceptor nuclei $(*)$ and outer

caspase-12 activity (see above) at day 1 supports this assumption, as ER stress has been shown to activate these caspases in retinal degeneration [23]. To gain further insight, we have quantified the gene expression of CHOP and GRP78, known to be upregulated under ER stress conditions. An increase in the mRNA level compared to control was found after MNU treatment (Fig. 6). CHOP mRNA expression was significantly upregulated at all time points PI compared to the control (fold upregulation on d1 PI: $4.7 \pm 0.6$, on d3 PI: $5.6 \pm 0.3$, on d7 PI: 5.6 $\pm 0.4 ; P<0.001)$ and reached a plateau on d3 PI. GRP78 was also significantly upregulated at all time points PI compared to the control (fold upregulation on d1 PI: $2.847 \pm 0.387$, on d3 PI: $3.516 \pm 0.698$, on d7 PI: $4.745 \pm 1.154 ; P<0.001$ ); the values increased over time with the highest expression on day 7 PI.

\section{Discussion}

Animal models of retinal diseases are an important tool for studying degenerative processes as well as for developing potential treatment strategies to interfere with the induced cell segments (\#) interchanged adjacent to the RPE monolayer (c). Subsequently, photoreceptor outer segments were completely missing, whereas the RPE monolayer remained intact (d; arrows show microvilli) at later time points. Panel e shows healthy mouse retina with an RPE cell layer, microvilli (arrows), photoreceptor outer segments (black arrowheads) and Bruch's membrane $\left(^{*}\right)$ for comparison

death. Pharmacological approaches, including MNU-induced degeneration, have the advantage of an arbitrary modulation of onset and severity. The effect of MNU on PR cells in mice has been investigated, and it is the common understanding that apoptosis is thereby induced in PRs [13]. This was also evident in our experimental setting, as the number of TUNEL-positive cells in the ONL increased significantly in response to MNU administration over time (Fig. 1). On the cellular level, PR outer segments were completely missing, whereas the RPE monolayer and Bruch's membrane remained intact. Subcellular changes were visualized using EM, and thereby chromatin condensation and apoptotic bodies were found exclusively in the ONL (Fig. 2). Cell shrinkage and pyknosis due to chromatin condensation are early signs of apoptosis [24]. Therefore, a photoreceptor-specific toxicity of MNU has been suggested $[10,12,25]$. However, in addition to falsepositive cells due to EM preparation procedures, these cells imaged by EM represent exclusively late apoptotic cells, making it impossible to clearly define the involved cell death pathway.

Therefore, we investigated the underlying PCD components of MNU-induced PR degeneration. Earlier publications 


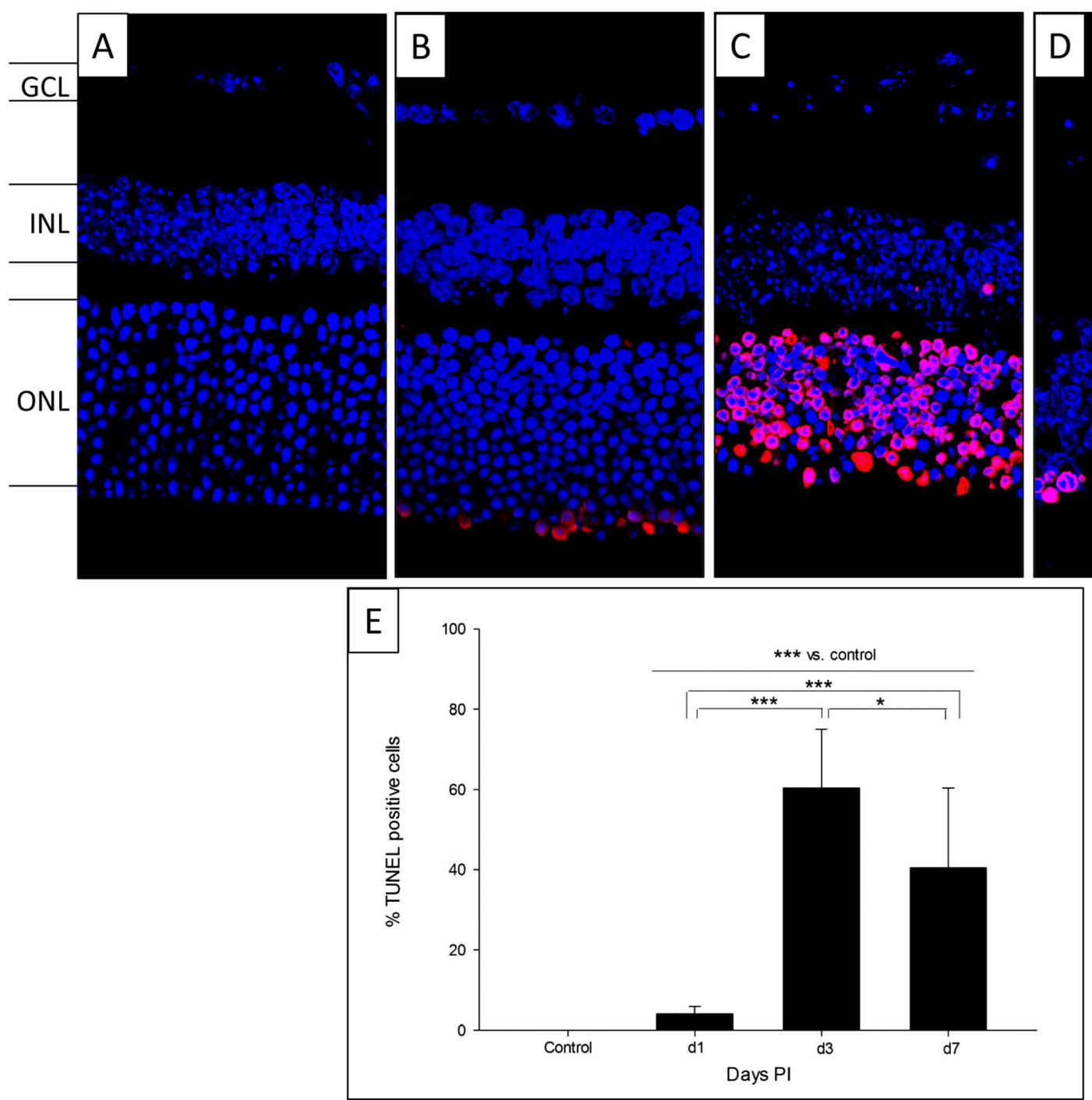

Fig. 3 TUNEL-positive cells after MNU treatment were only detected in the outer nuclear layer (ONL). Whereas no TUNEL-positive cells could be found in the control samples (a) TUNEL-positive cells (red) were detected beginning at day 1 PI (b). The maximum of TUNEL positivity was found 3 days PI (c). As only about 1-2 rows of photoreceptor nuclei remain 7 days PI, the number of TUNEL-positive cells decreased at that time point (d). The scale bar equals $20 \mu \mathrm{m}$. The graph in e depicts the quantification of TUNEL-positive cells after MNU treatment $(* * * P \leq$ $0.001 ; * P \leq 0.05)$ by us and others have pointed towards caspase-3-dependent and/or caspase-3-independent mechanisms [10, 26, 27]. Herein, we measured the significant upregulation of a cascade of proteases involved in cell death execution. Thereby, the activity of caspase-3, and to a lesser degree of caspases-9 and 12, was found to be increased (Fig. 4). However, the detected values were relatively small compared to the substantial amount of PR cell death observed by TUNEL staining (Fig. 3), indicating the contribution of other nonconventional cell death pathways. The relatively low expression of caspase3 might also explain the nondetectability of this caspase in immunohistochemistry-only approaches [10]. Nevertheless, MNU has been shown to increase caspase-3 mRNA expression in the retina of MNU-treated rats [28]. Consistently, a caspase-3 inhibitor decreased the loss of photoreceptors in Sprague-Dawley rats after MNU treatment [5].

As several studies have supported the idea of the contribution of calpains (calcium-dependent proteases) in degenerative processes, we have also quantified the level of calpain activity after MNU treatment. Calpains, ubiquitously expressed and activated depending on the concentration of calcium present, have been detected in PR cell death in $r d l$ mice [16] as well as in $\mathrm{P} 23 \mathrm{H}$ and S334ter rhodopsin mutant rats [17]. Thereby, increased calcium levels are released from the ER or the mitochondria in response to toxic insult. In our samples, a significant upregulation of calpain activity in the MNU samples and colocalization of TUNEL- and calpainpositive cells in the ONL was also found (Fig. 5). 
Fig. 4 Activity assays for key caspases in PCD. Graphs depict the time course of caspase- $3,-9$, and -12 activities with and without MNU treatment.

Sequential upregulation of initiators and effectors of PCD is seen. Error bars indicate the SD from three independent experiments, and statistically significant differences are indicated with an asterisk $(P \leq$ $0.01)$. Note the different scale on the $y$-axes
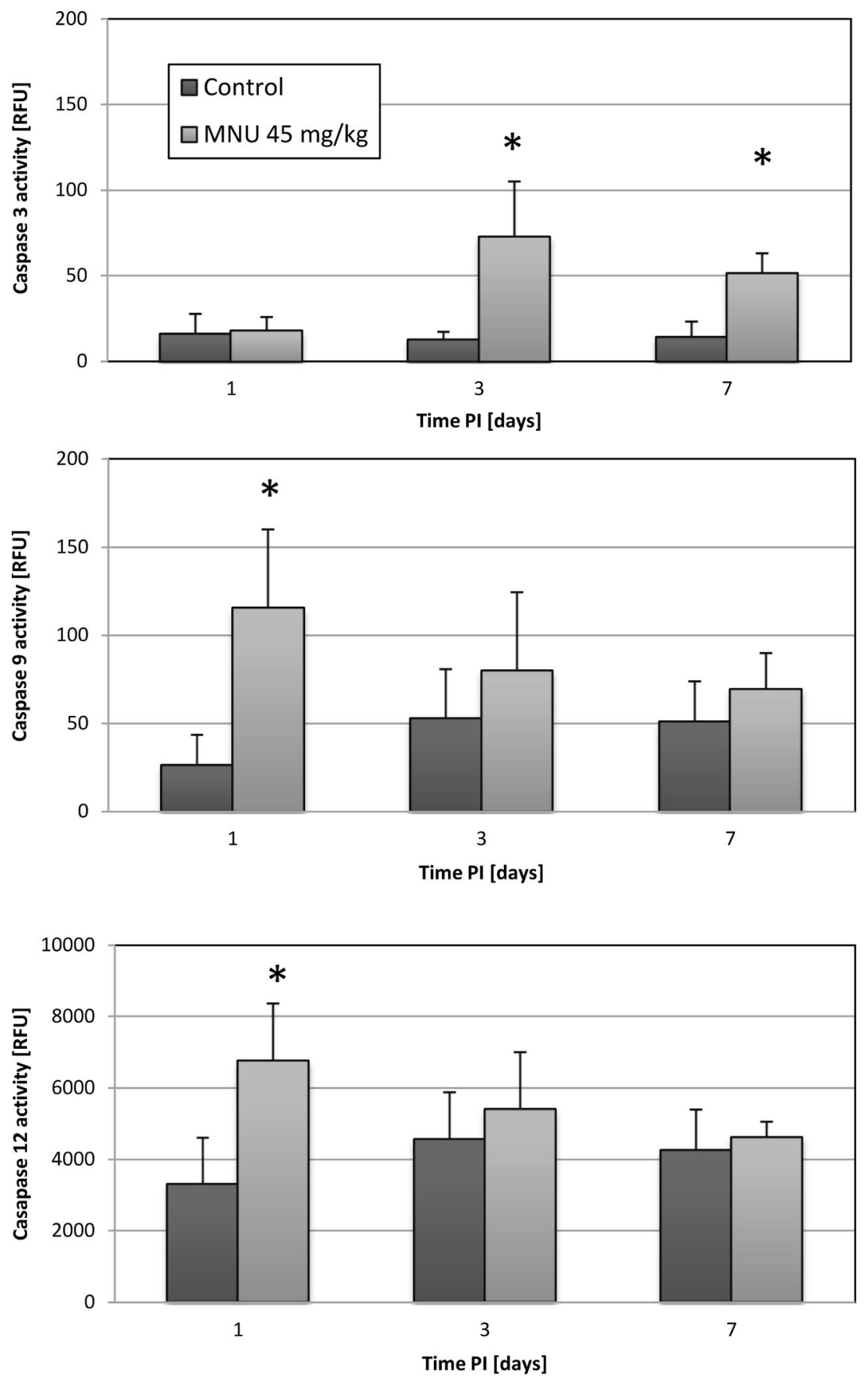

Nevertheless, compared to the high amount of TUNELpositive cells, we detected only a low number of cells displaying calpain activity. However, calpain activity was also present in samples obtained from wild type retinas. This result can be explained by occasional apoptotic events in the ONL, since photoreceptors are capable of executing developmental apoptosis at least until P42, as shown by Arango-Gonzalez and colleagues [29]. Our results are consistent with previous studies showing that the oral administration or i.p. delivery of a calpain inhibitor reduced but did not fully rescue the MNU- induced loss of photoreceptors [26, 30]. However, we cannot exclude the involvement of additional cell death pathways (e.g., PARP).

Caspase-12 plays a central role in ER stress-mediated apoptosis [31]. Upon activation by ER stress it translocates from the ER to the cytosol, where it directly cleaves pro-caspase-9, which, in turn, activates the effector caspase, caspase-3 [32]. The upregulation of this specific caspase cascade in our samples indicates an involvement of ER-related stress in MNU-induced 

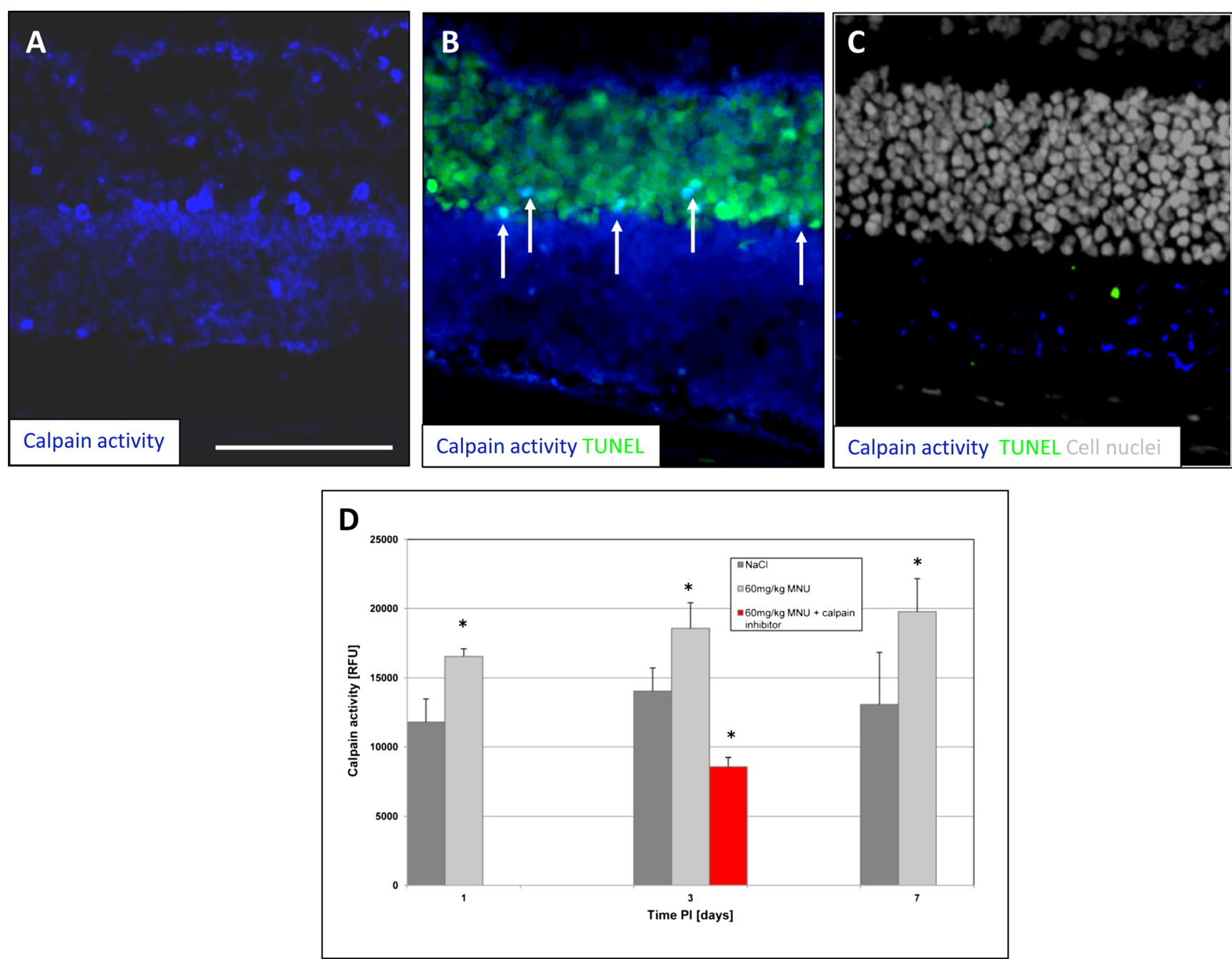

Fig. 5 Activity assays for calpain. Images depict in-situ staining of cells positive for active calpain (a, blue) at day 3 PI. Panel b shows TUNELpositive cells (green) and calpain/TUNEL-positive cells (arrows) in the ONL, indicating that the MNU-induced PCD involves calpain activation. Staining of the control retinas displayed does not depict such doublepositive cells (c). The scale bar represents $50 \mu \mathrm{m}$. The graph in panel d reflects the quantification of calpain activity with and without MNU treatment over time. Significant upregulation has been measured at all time points, whereas adding the calpain inhibitor Z-LLY-FMK led to a significant decrease in the level of activated calpain (day 3 PI). Error bars indicate the SD from three independent experiments, and statistically significant differences are indicated with an asterisk $(P \leq 0.01)$
PCD. Furthermore, we were also able to detect CHOP and GRP78 on the transcriptional level after MNU treatment. CHOP is known to be induced in response to ER stress [33], and activates Bim, a Bcl-2 family member, which is required for ER-stress mediated apoptosis [34]. Whereas CHOP mRNA expression was highly upregulated from day 1 PI and stayed elevated until 7 days PI, GRP78 expression increased continuously over time (Fig. 6). Dying photoreceptors and their secreted factors might trigger stress response in other retinal cells. In this regard, the measured results, especially at day 7 where only approx. $10 \%$ PR remain, represent the stress response from the remaining photoreceptors and adjacent retinal cells. Elevated GRP78 mRNA levels are a sensitive indicator of ER stress, and have been observed in diseases linked to ER stress [35] and in retinal degeneration models arising from P23H rhodopsin expression [36]. During ER stress, there is also an accumulation of misfolded proteins within the ER due to either primary (genetic) or secondary (environmental) factors [37]. Although the analysis of the unfolded protein response was not in the scope of this manuscript, our findings may suggest that this closely related pathway also participates in MNUinduced PR cell death.

In conclusion, regular apoptosis is the prevalent cause of MNU-induced PR cell death. However, involvement of alternative PCD pathways including ER stress and calpain activation was also found (Table 1), indicating that the sole use of caspase inhibitors will not be 


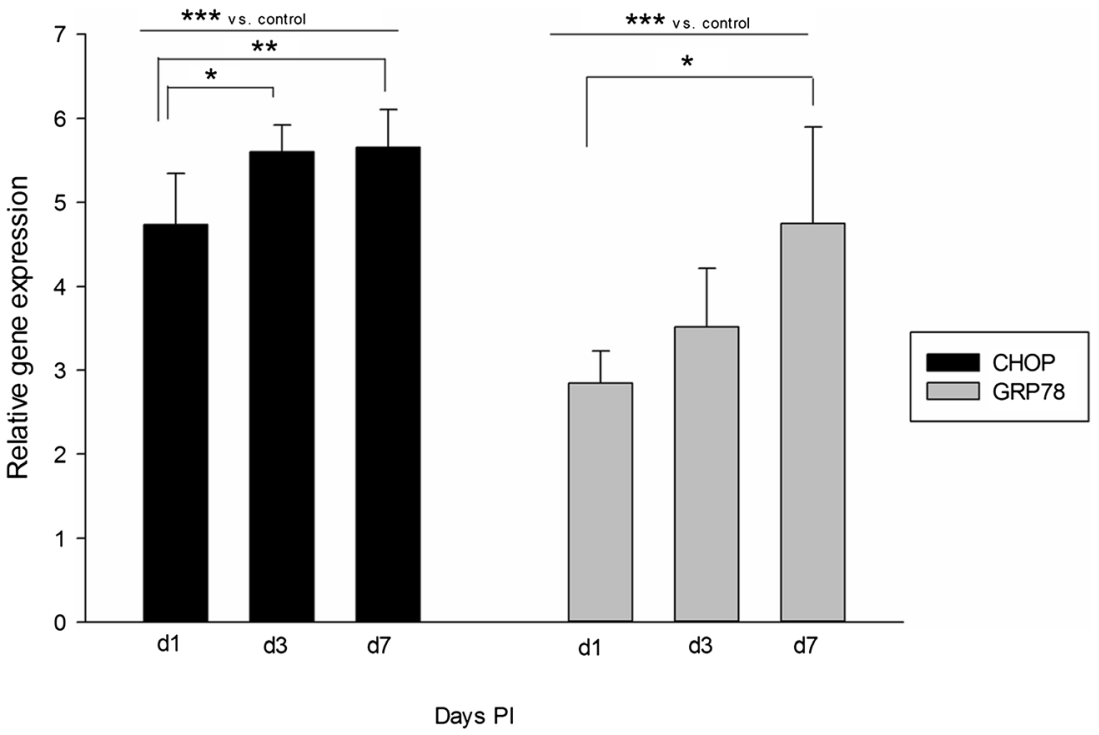

Fig. 6 qPCR analysis of relative gene expression of CHOP. Values were normalized against the reference gene Gapdh and control samples. Expression in control samples was set to ' 1 '. CHOP and GRP78 expression was significantly increased $(* * * P \leq 0.001)$ in MNU samples compared to control samples at all time points PI. As a result, CHOP

expression levels remained steadily elevated at time points $d 3$ and $d 7$, but were significantly increased $(* P \leq 0.05 / * * P \leq 0.005)$ when compared to $d 1$. On the other hand, GRP78 expression increased continuously over the investigated time, with significant higher levels on $d 7$ PI compared to $d l$ PI $(* P \leq 0.05)$

sufficient to prevent MNU-induced photoreceptor cell death. It seems to be a common feature that several cell death pathways add to the degenerative process, and all of them would need to be inhibited to restore visual function. Thereby, the need for multimodal treatments to gain positive effects might be even more reflective of the in-situ situation of human diseases. In this regard, the MNU model could serve as a helpful tool to investigate such treatment options targeting the survival of neuronal cells in the degenerated retina. Another issue is the fact that the inhibition of one cell death pathway might trigger the activation of one or several others, further aggravating the course of the

disease. Additionally, the severity of the damage might influence the involved cell death mechanisms. The use of appropriate concentrations of MNU provides a means of actively manipulating the duration and extent of photoreceptor degeneration. That might be usable to monitor possible beneficial effects after neuroprotective treatment or cell-based regenerative approaches. Nevertheless, MNU is a highly toxic and labile substance, demanding careful handling. As the specific mechanism of MNU action in the neuroretina is only partially known, investigation of this mechanism should be intensified in order to use the entire potential of the model.

Table 1 Summary of the involvement of different PCD pathways in MNU-induced retinal degeneration

\begin{tabular}{|c|c|c|c|c|c|c|}
\hline & $\begin{array}{c}\text { Caspase-3 } \\
\text { activity }\end{array}$ & $\begin{array}{c}\text { Caspase-9 } \\
\text { activity }\end{array}$ & $\begin{array}{c}\text { Caspase-12 } \\
\text { activity }\end{array}$ & $\begin{array}{l}\text { Calpain } \\
\text { activity }\end{array}$ & $\begin{array}{l}\text { CHOP } \\
\text { mRNA }\end{array}$ & $\begin{array}{l}\text { CHOP } \\
\text { Protein }\end{array}$ \\
\hline $\mathrm{D} 1 \mathrm{PI}$ & n.d. & 1 & $\overline{1}$ & & & n.d. \\
\hline D3 PI & t & \pm & \pm & & $\bar{t}$ & n.d. \\
\hline D7 PI & $\mathbf{t}$ & \pm & n.d. & 1 & $t$ & $\overline{1}$ \\
\hline
\end{tabular}

significant upregulation; \pm nonsignificant upregulation; $n . d$. no difference compared to control 
Acknowledgments The authors wish to thank Agathe Duda, Monika Kilchenmann, Anelia Schweri-Olac and Beat Haenni for their excellent technical assistance. This work was partly supported by the Fritz Tobler Foundation and the Bern University Research Foundation.

\section{References}

1. Tsubura A, Yoshizawa K, Kuwata M, Uehara N (2010) Animal models for retinitis pigmentosa induced by MNU: disease progression, mechanisms and therapeutic trials. Histol Histopathol 25:933944

2. Christmann M, Kaina B (2000) Nuclear translocation of mismatch repair proteins MSH2 and MSH6 as a response of cells to alkylating agents. J Biol Chem 275:36256-36262

3. Jobst K (1967) Teratogenous changes and tumors in rats following treatment with methylnitroso-urea (MNU). Neoplasma 14:435-436

4. Herrold KM (1967) Pigmentary degeneration of the retina induced by $\mathrm{N}$-methyl-N-nitrosourea. An experimental study in Syrian hamsters. Arch Ophthalmol 78:650-653

5. Yoshizawa K, Yang J, Senzaki H, Uemura Y, Kiyozuka Y, Shikata N, Oishi Y, Miki H, Tsubura A (2000) Caspase-3 inhibitor rescues Nmethyl-N-nitrosourea-induced retinal degeneration in SpragueDawley rats. Exp Eye Res 71:629-635

6. Petrin D, Baker A, Coupland SG, Liston P, Narang M, Damji K, Leonard B, Chiodo VA, Timmers A, Hauswirth W, Korneluk RG, Tsilfidis C (2003) Structural and functional protection of photoreceptors from MNU-induced retinal degeneration by the X-linked Inhibitor of apoptosis. Invest Ophthalmol Vis Sci 44:2757-2763

7. Doonan F, Donovan M, Cotter TG (2003) Caspase-independent photoreceptor apoptosis in mouse models of retinal degeneration. $\mathrm{J}$ Neurosci 23:5723-5731

8. Nakajima M, Nambu H, Shikata N, Senzaki H, Miki H, Tsubura A (1996) Pigmentary degeneration induced by N-methyl-N-nitrosourea and the fate of pigment epithelial cells in the rat retina. Pathol Int 46: 874-882

9. Hisatomi T, Sakamoto T, Sonoda K, Tsutsumi C, Qiao H, Enaida H, Yamanaka I, Kubota T, Ishibashi T, Kura S, Susin SA, Kroemer G (2003) Clearance of apoptotic photoreceptors. Am J Pathol 162: 1869-1879

10. Zulliger R, Lecaudé S, Eigeldinger-Berthou S, Wolf-Schnurrbusch UEK, Enzmann V (2011) Caspase-3-independent photoreceptor degeneration by $\mathrm{N}$-methyl-N-nitrosourea (MNU) induces morphological and functional changes in the mouse retina. Graefe's Arch Clin Exp Ophthalmol 249:859-869

11. Chen YY, Liu SL, Hu DP, Xing YQ, Shen Y (2014) N-methyl-Nnitrosourea-induced retinal degeneration in mice. Exp Eye Res 121: $102-113$

12. Yuge K, Nambu H, Senzaki H, Nakao I, Miki H, Uyama M, Tsubura A (1996) N-methyl-N-nitrosourea-induced photoreceptor apoptosis in the mouse retina. In Vivo 10:483-488

13. Mordes D, Luo X, Kar A, Kuo D, Xu L, Fushimi K, Yu G, Sternberg $\mathrm{P} J \mathrm{~J}$, Wu JY (2006) Pre-mRNA splicing and retinitis pigmentosa. Mol Vis 12:1259-1271

14. Tsubura A, Yoshizawa K, Kuro M (2013) N-methyl-N-nitrosourea animal models for retinitis pigmentosa. In: Conn PM (ed) Animal models for the study of human disease, 1st edn. Academic, Boston, pp $117-142$

15. Lohr HR, Kuntchithapautham K, Sharma AK, Rohrer B (2006) Multiple, parallel cellular suicide mechanisms participate in photoreceptor cell death. Exp Eye Res 83:380-389

16. Paquet-Durand F, Azadi S, Hauck SM, Ueffing UM, van Veen T, Ekström P (2006) Calpain is activated in degenerating photoreceptors in the rd1 mouse. J Neurochem 96:802-814
17. Kaur J, Mencl S, Sahaboglu A, Farinelli P, van Theo V, Zrenner E, Ekstrom P, Paquet-Durand F, Aarango-Gonzalez B (2011) Calpain and PARP activation during photoreceptor cell death in $\mathrm{P} 23 \mathrm{H}$ and S334ter rhodopsin mutant rats. PLoS ONE 6:e22181

18. Yang LP, Wu LM, Guo XJ, Li Y, Tso MO (2008) Endoplasmic reticulum stress is activated in light-induced retinal degeneration. $\mathrm{J}$ Neurosci Res 86:910-919

19. Kaufman RJ (1999) Stress signaling from the lumen of the endoplasmic reticulum: coordination of gene transcriptional and translational controls. Genes Dev 13:1211-1233

20. Kroeger H, Messah C, Ahern K, Gee J, Joseph V, Matthes MT, Yasumura D, Gorbatyuk MS, Chiang WC, LaVail MM, Lin JH (2012) Induction of endoplasmic reticulum stress genes, BiP and Chop, in genetic and environmental models of retinal degeneration. Invest Ophthalmol Vis Sci 53:7590-7599

21. Malhi H, Kropp EM, Clavo VF, Kobrossi CR, Han JS, Mauer AS, Yong J, Kaufman RJ (2013) C/EBP homologous protein-induced macrophage apoptosis protects mice from steatohepatitis. J Biol Chem 288:18624-18642

22. Gomez-Vicente V, Donovan M, Cotter TG (2005) Multiple death pathways in retina-derived $661 \mathrm{~W}$ cells following growth factor deprivation: crosstalk between caspases and calpains. Cell Death Differ 12:796-804

23. Sanges D, Comitato A, Tammaro R, Marigo V (2006) Apoptosis in retinal degeneration involves cross-talk between apoptosis-inducing factor (AIF) and caspase-12 and is blocked by calpain inhibitors. Proc Natl Acad Sci U S A 103:17366-17371

24. Kerr JF, Wyllie AH, Currie AR (1972) Apoptosis: a basic biological phenomenon with wide-ranging implications in tissue kinetics. $\mathrm{Br} \mathrm{J}$ Cancer 26:239-257

25. Rösch S, Johnen S, Mataruga A, Muller F, Pfarrer C, Walter P (2014) Selective photoreceptor degeneration by intravitreal injection of Nmethyl-N-nitrosourea. Invest Ophthalmol Vis Sci 55:1711-1723

26. Oka T, Nakajima T, Tamada Y, Shearer TR, Azuma M (2007) Contribution of calpains to photoreceptor cell death in N-methyl-Nnitrosourea-treated rats. Exp Neurol 204:39-48

27. Yoshizawa K, Nambu H, Yang J, Oishi Y, Senzaki H, Shikata N, Miki H, Tsubura A (1999) Mechanisms of photoreceptor apoptosis induced by N-Methyl-N-nitrosourea in Sprague-Dawley rats. Lab Investig 79:1359-1367

28. Wang D, Wang Z, Li Y, Chen X, Sun GY (2013) Nimodipine inhibits $\mathrm{N}$-methyl-N-nitrosourea-induced retinal photoreceptor apoptosis in vivo. Indian J Pharmacol 45:149-154

29. Arango-Gonzalez B, Trifunovic D, Sahaboglu A, Kranz K, Michalakis S, Farinelli P, Koch S, Koch F, Cottet S, JanssenBienhold U, Biel M, Zrenner E, Euler T, Ekstrom P, Ueffing M, Paquet-Durand F (2014) Identification of a common non-apoptotic cell death mechanism in hereditary retinal degeneration. PLoS ONE 9:e112142

30. Kuro M, Yoshizawa K, Uehara N, Miki H, Takahashi K, Tsubura A (2011) Calpain inhibition restores basal autophagy and suppresses MNU-induced photoreceptor death in mice. In Vivo 25:617-624

31. Szegezdi E, Fitzgerald U, Samali A (2003) Caspase-12 and ERstress-mediated apoptosis: the story so far. Ann N Y Acad Sci 1010:186-194

32. Morishima N, Nakanishi K, Takenouchi H, Shibata T, Yasuhiko Y (2002) An endoplasmic reticulum stress-specific caspase cascade in apoptosis. Cytochrome c-independent activation of caspase- 9 by caspase-12. J Biol Chem 277:34287-34294

33. Wang XZ, Lawson B, Brewer JW, Zinszner H, Sanjay A, Mi LJ, Boorstein R, Kreibich G, Hendershot LM, Ron D (1996) Signals from the stressed endoplasmic reticulum induce C/EBP-homologous protein (CHOP/GADD153). Mol Cell Biol 16:4273-4280

34. Puthalakath H, O'Reilly LA, Gunn P, Lee L, Kelly PN, Huntington ND, Hughes PD, Michalak EM, Kimm-Breschkin J, Motoyama N, 
Gotoh T, Akira S, Bouillet P, Strasser A (2007) ER stress triggers apoptosis by activating BH3-only protein Bim. Cell 129:1337-1349

35. Lindholm D, Wootz H, Korhonen L (2006) ER stress and neurodegenerative diseases. Cell Death Differ 13:385-392

36. Gorbatyuk MS, Knox T, LaVail MM, Gorbatyuk OS, Noorwez SM, Hauswirth WW, Lin JH, Muzyczka N, Lewin AS (2010) Restoration of visual function in $\mathrm{P} 23 \mathrm{H}$ rhodopsin transgenic rats by gene delivery of BiP/Grp78. Proc Natl Acad Sci U S A 107:5961-5966

37. Kaser A, Blumberg RS (2011) Autophagy, microbial sensing, endoplasmic reticulum stress, and epithelial function in inflammatory bowel disease. Gastroenterology 140:17381747 